\title{
ANÁLISE CRÍTICA DOS INDICADORES DE DESEMPENHO DE ESTAÇÕES DE TRATAMENTO DE EFLUENTES DO ÓRGÃO AMBIENTAL ESTADUAL DO RIO DE JANEIRO
}

\author{
Waldyr Ramos Junior \\ waldyrjunior@id.uff.br \\ Universidade Federal Fluminense - \\ UFF, Niterói, Rio de Janeiro, Brasil.
}

\section{Lívia Maria da Costa Silva}

liviamaria@id.uff.br

Universidade Federal Fluminense UFF, Niterói, Rio de Janeiro, Brasil.

\section{RESUMO}

A avaliação de desempenho das estações de tratamento de efluentes (ETE), no geral, se baseia em aferir apenas a qualidade do efluente final, não se levando em consideração outros parâmetros, que também contribuem substancialmente para o desempenho. Nesse sentido, indicadores são ferramentas importantes, por serem capazes de resumir em um único valor o estado do objeto avaliado, considerando seus diversos aspectos e características. Objetiva-se, com esse trabalho, pontuar a importância de indicadores de desempenho para avaliação e licenciamento de ETE, além de avaliar a utilização dos indicadores de qualidade de ETE estabelecidos por meio de duas normas institucionais (NOI-INEA 11 e NOI-INEA 14) elaboradas pelo Instituto Estadual do Ambiente (INEA), órgão ambiental do estado do Rio de Janeiro. Realizou-se uma revisão bibliográfica em artigos referentes à temática de indicadores de desempenho de ETE, juntamente com uma pesquisa nos sites de todos os órgãos ambientais estaduais e distrital. Ademais, para análise crítica, adotou-se a metodologia de pesquisa de documentos oficiais no acervo digital do INEA. Com isso, foi possível perceber que, no Brasil, a existência de legislações que utilizem indicadores para avaliação de ETE ainda é restrito ao aludido estado. No entanto, apesar de pouco utilizados, os indicadores apresentam grande potencial para auxiliar no licenciamento ambiental e na fundamentação de pareceres técnicos, contribuindo para uma melhor informação e participação da sociedade.

Palavras-chaves: Licenciamento ambiental; Gestão ambiental; Órgãos ambientais estaduais; Qualidade ambiental. 


\section{INTRODUÇÃO}

As estações de tratamento de efluentes (ETE) têm como principal objetivo promover a depuração dos efluentes, sejam eles de origem doméstica ou industrial, antes do seu descarte final, geralmente em corpos hídricos. Com isso, almeja-se implementar tecnologias cada vez mais eficientes na remoção da carga poluidora do efluente e, se possível, com a geração de poucos resíduos e rejeitos.

No entanto, surge a necessidade de avaliar o desempenho dessas estações, de forma mais holística, para além do atendimento dos padrões de lançamento estipulados na legislação ambiental. Segundo Raschle (2013), a avaliação de desempenho é uma importante ferramenta para identificar deficiências de operação, segurança e meio ambiente de ETE.

Para Piza e Paganini (2006), indicadores são instrumentos de gestão pelos quais se medem o desempenho e o funcionamento de um serviço ou unidade produtiva, por meio do qual é possível identificar melhorias necessárias, tais como adequações e/ou ampliações.

A Agência Reguladora de Águas, Energia e Saneamento do Distrito Federal (ADASA, 2016, p. 37) conceitua indicadores de desempenho como: "índices que traduzem de modo sintético os aspectos mais relevantes do desempenho, em geral, operacional e econômico-financeiro de uma entidade, simplificando a sua análise".

Dessa forma, indicadores podem ser definidos como uma ferramenta de informações quantificadas com a capacidade de sintetizar e compilar uma série de características do ambiente ou fenômeno avaliado. Os indicadores informam dados sobre o estado do fenômeno ou meio, de fácil compreensão, o que possibilita a sua utilização na tomada de decisão e na transmissão de informação para toda a sociedade.

Neste mesmo caminho, o Ministério do Meio Ambiente (MMA, 2019) define os indicadores ambientais como estatísticas selecionadas que representam ou resumem alguns aspectos do estado do meio ambiente, dos recursos naturais e de atividades humanas relacionadas. Sendo assim, a utilização de um indicador ambiental parece ser uma promissora alternativa na avaliação de desempenho das ETE, considerando que a utilização de indicadores de desempenho ambiental já é amplamente realizada nas indústrias ao redor do mundo (Melo; Souza, 2014). Além disso, por meio dos resultados de indicadores é possível realizar uma avaliação da evolução do desempenho no tempo, bem como analisar comparativamente algumas organizações do setor, inclusive de outras regiões (ADASA, 2016).
Portanto, o objetivo deste artigo é avaliar a utilização e a relevância dos indicadores de qualidade de estações de efluentes estabelecidos por meio de duas normas institucionais (NOI-INEA 11 e NOI-INEA 14) elaboradas pelo Instituto Estadual do Ambiente (INEA), órgão ambiental do estado do Rio de Janeiro.

\section{MÉTODO}

No presente artigo, inicialmente, realizou-se uma revisão bibliográfica em artigos referentes à temática de tratamento de efluentes, por meio da qual foram selecionados três artigos nacionais (Sperling; Sperling, 2013; Barros, 2013; de Paula, 2013) e três artigos internacionais (Lindtner et al., 2008; Balmér; Hellström, 2012; ERSAR, 2020) como base deste estudo. A pesquisa foi realizada no período de junho de 2018 a março de 2020, utilizando as palavras-chave: indicadores de desempenho, estações de tratamento de esgoto, indicadores de qualidade, e estações de tratamento de despejos industriais. A busca foi realizada nos principais portais de periódicos, como o da CAPES e SciELO. Utilizou-se como critério de seleção o enquadramento nas palavras-chave e relevância para o presente artigo. Ademais, foi realizada uma pesquisa nos sites dos órgãos ambientais estaduais brasileiros e do Distrito Federal e em países de destaque internacional com produção cientifica na área de indicadores de desempenho de estações de tratamento de esgoto.

No contexto do estado do Rio de Janeiro, adotou-se a metodologia de pesquisa de documentos oficiais (normativas internas, pareceres técnicos, atas de reuniões do Conselho Diretor do Órgão) no acervo digital do órgão ambiental estadual - INEA, buscando por IQE (Índice de Qualidade de Operação de Estação de Tratamento de Esgoto), IQETDI (Índice de Qualidade de Operação de Estação de Tratamento de Despejos Industriais), Índice de Qualidade de Estações de tratamento, além da realização de reuniões com os responsáveis pelo setor de licenciamento de ETE e troca de e-mails com o principal técnico idealizador dos índices neste órgão.

\section{RESULTADOS E DISCUSSÃO}

\section{Indicadores de desempenho de estações de tratamento de efluentes no âmbito internacional}

Conforme diagnosticado por Barros (2013), existem diversas organizações no âmbito internacional que possuem um sistema de indicadores de desenvolvimento sobre o sistema de saneamento. No entanto, poucos são os sistemas de indicadores específicos para estações de 
tratamento de esgoto, destacando-se os indicadores da Austrian Water and Waste Association (AWWA - Associação Austríaca de Água e Resíduo), do Laboratório Nacional de Engenharia Civil de Portugal (LNEC) e da Swedish Water \& Wastewater Association (SWWA - Associação Sueca de Água e Esgoto).

A AWWA desenvolveu o sistema austríaco de benchmarking durante seis anos (1999-2004) e teve como principais objetivos: o desenvolvimento de indicadores de desempenho e identificar bons desempenhos e potenciais reduções de custo de ETE (Lindtner et al., 2008). Conforme observado por de Paula (2013), o indicador de desempenho da AWWA é classificado em três categorias principais: financeira, técnica e processos. O desempenho do sistema é obtido a partir da correlação entre os indicadores de processos com os indicadores técnicos.

O LNEC desenvolveu um projeto chamado PASt21 que é uma Iniciativa Nacional de Avaliação de Desempenho de Estações de Tratamento de Água (ETA) e de Esgoto (ETE) urbanas e tem como objetivo estabelecer a avaliação de desempenho e o benchmarking, e reforçar a aplicabilidade do sistema de avaliação de desempenho das aludidas estações, inclusive de diferentes tipologias. 0 indicador de desempenho analisa oito domínios da estação: a qualidade da água residual tratada; a eficiência e a confiabilidade; a utilização de água, a energia e os materiais; a gestão de subprodutos; a segurança; os recursos humanos; os recursos econômico-financeiros; e o apoio ao planejamento e projeto.

O sistema de indicadores criado pela SWWA, em 2003, em uma plataforma na internet chamada VASS, com o início da coleta de dados de estações de tratamento de esgoto da Suécia em 2009, teve a finalidade de propiciar a comparação entre as ETE do país e de outros países, por meio de indicadores, possibilitando um benchmarking entre as estações. A avaliação das ETE envolve as seguintes dimensões: qualidade dos efluentes, lodo, energia, produtos químicos e aspectos econômicos (Balmér; Hellström, 2012).

É importante destacar uma característica comum nos objetivos dos sistemas de indicadores supracitados, que é o benchmarking. Tal prática é possível, porque a maioria das ETE são de gestão pública, e possibilita o compartilhamento de boas práticas e casos de sucesso.

\section{Indicadores de desempenho de estações de tratamento de efluentes no âmbito nacional}

A Lei do Saneamento Básico, Lei no 11.445/2007 (Brasil, 2007), dispõe em seu artigo 23 sobre os aspectos mí- nimos da avaliação da prestação de serviços de saneamento, dos quais valem destacar os seguintes: padrões e indicadores de qualidade da prestação dos serviços; metas progressivas de expansão e de qualidade dos serviços e os respectivos prazos; monitoramento dos custos; avaliação da eficiência e eficácia dos serviços prestados; padrões de atendimento ao público e mecanismos de participação e informação.

Neste sentido, é imperioso que o Brasil também tenha indicadores capazes de avaliar as ETE, de uma forma mais abrangente do que apenas no quesito de atendimento aos padrões de lançamento de efluentes preconizados na legislação, uma vez que o atendimento à legislação ambiental pode não assegurar a proteção integral da qualidade ambiental dos corpos receptores de efluentes (Bertoletti, 2015).

Além disso, há também um benefício para a saúde pública, de uma maneira geral, haja vista que atualmente, no Brasil, as doenças de vinculação hídrica, como cólera, febres tifoide e paratifoide, amebíase, diarreia e esquistossomose são responsáveis por um elevado número de internações. Segundo o Instituto Trata Brasil (2019), a incidência de internações por doenças associadas à falta de saneamento foi de 12,46 internações por $10 \mathrm{mil}$ habitantes.

Atualmente, no país, existem indicadores para avaliar o esgotamento, onde, talvez, o mais conhecido seja o Sistema Nacional de Informação sobre Saneamento (SNIS). Tal sistema possui abrangência nacional e realiza a coleta de dados de caráter institucional, administrativo, operacional, gerencial, econômico-financeiro, contábil e de qualidade da prestação de serviços de saneamento básico em áreas urbanas dos quatro componentes do saneamento básico (água, esgoto, resíduos sólidos e drenagem e manejo de águas pluviais).

O SNIS tem os seguintes objetivos: (i) planejamento e execução de políticas públicas; (ii) orientação da aplicação de recursos; (iii) conhecimento e avaliação do setor saneamento; (iv) avaliação de desempenho dos serviços; (v) aperfeiçoamento da gestão; (vi) orientação de atividades regulatórias e de fiscalização; e (vii) exercício do controle social.

Outras organizações também dispõem de uma proposta de indicadores para avaliar o sistema de esgotamento, dentre elas pode-se destacar a Associação Brasileira de Agências de Regulação (ABAR), Agência Reguladora de Serviços Públicos Delegados do Estado do Ceará (ARCE) e ADASA.

No entanto, esses sistemas de indicadores se direcionam para avaliar o desempenho dos sistemas de esgota- 
mento de uma forma mais global, compreendendo outras partes além das ETE, como rede coletora e relação de satisfação do cliente, por exemplo. Nessa questão, no Brasil, existem poucos indicadores de desempenho que se dediquem exclusivamente à avaliação de ETE, podendo citar os indicadores propostos por Barros (2013) para avaliação das ETE de Brasília - DF e o sistema proposto por de Paula (2013).

Neste contexto, vale trazer a informação da existência do Prêmio Nacional de Qualidade em Saneamento (PNQS) da Associação Brasileira de Engenharia Sanitária e Ambiental (ABES). Anualmente, premiam-se as companhias de serviço em saneamento que apresentam melhor desempenho.

A avaliação das companhias é feita por meio de indicadores de desempenho, publicados anualmente no Guia de Referência para Medição do Desempenho (GRMD) e a lista dos indicadores é dividida em cinco grupos: econômico-financeiros, sociais e ambientais, relativos aos clientes e mercado, relativos às pessoas e relativos ao processo.

Nas últimas edições, as companhias premiadas têm sido do Sudeste, podendo-se destacar a Companhia de Saneamento Básico do Estado de São Paulo (SABESP) e a Companhia de Saneamento de Minas Gerais (COPASA). Nas demais regiões do país, destacam-se, no Sul: Companhia Riograndense de Saneamento (CORSAN) e Companhia de Saneamento do Paraná (SANEPAR); no Nordeste: Companhia de Saneamento de Sergipe (DESO) e Empresa Baiana de Águas e Saneamento (EMBASA); e no Centro-Oeste: Companhia Saneamento de Goiás (SANEAGO).

Indicadores de desempenho de estações de tratamento de efluentes no estado do Rio de Janeiro

No contexto do estado do Rio de Janeiro, o INEA foi criado em 2007, com a missão de integrar a política ambiental estadual, sendo um órgão gestor ambiental de referência, exercendo um papel estratégico no desenvolvimento estadual.

Em 2015, por meio do Conselho Diretor (CONDIR), órgão máximo do INEA e composto pelos titulares da Presidência e Diretorias do Instituto, houve a aprovação de duas normas institucionais (NOI), a NOI-INEA 11 (INEA, 2019) e a NOI-INEA 14 (INEA, 2019), que visam normatizar e amparar a metodologia de cálculo do IQE e de cálculo do IQETDI no estado do Rio de Janeiro, respectivamente.

Os aludidos índices são calculados a partir de uma série de itens referentes à estrutura, qualidade do efluente tratado e conformidade legal e para o resultado de cada item é atribuído um peso. Um peso para um resultado positivo e outro para um resultado negativo e, em alguns casos, é atribuído um outro peso para um resultado intermediário. Os resultados são obtidos por meio de uma avaliação percentual de atendimentos aos critérios e do índice que varia de zero a dez, sendo classificado conforme demonstrado na Tabela 1.

Tabela 1. Avaliação do enquadramento do IQE e IQETDI

\begin{tabular}{|c|c|}
\hline $\begin{array}{c}\text { Enquadramento (IQE e } \\
\text { IQETDI) }\end{array}$ & Avaliação \\
\hline $0,0-6,0$ & Condições Inadequadas \\
\hline $6,1-8,0$ & Condições Regulares \\
\hline $8,1-10,0$ & Condições Adequadas \\
\hline \multicolumn{2}{|c|}{ Fonte: Autor (2020) }
\end{tabular}

Com isso, o órgão ambiental delineia e diferencia a avaliação da qualidade de operação da estação pela característica majoritária do efluente a ser tratado, ou seja, segundo a sua origem - doméstica ou industrial.

A criação de um índice de avaliação de estações de tratamento de efluentes coloca o INEA como pioneiro deste assunto, pois não foi encontrada nenhuma legislação neste mérito em nenhum outro órgão ambiental estadual e distrital. Ademais, nem mesmo o órgão ambiental federal, Instituto Brasileiro do Meio Ambiente e dos Recursos Naturais Renováveis (IBAMA) possui uma legislação nesse quesito.

Com isso, percebe-se que o INEA possui uma ferramenta muito importante para avaliar as estações. No entanto, desde a publicação dos índices (IQE e IQETDI), existem poucos registros da aplicação deles em pareceres técnicos elaborados pelo órgão, conforme busca realizada no site institucional. Este fato foi confirmado em entrevista com o chefe de serviço e com a analista ambiental responsável por essa área dentro do órgão (Gerência de Licenciamento de Atividades Não Industriais - GELANI). Verificou-se que o IQE já foi utilizado em um parecer técnico de indeferimento de um processo de renovação de licença de operação de uma estação de tratamento de esgoto doméstico da Companhia Estadual de Águas e Esgoto do Rio de Janeiro (CEDAE) (INEA, 2018).

Os indicadores têm grande versatilidade, pois esses têm o potencial de avaliar além da qualidade das estações, a sua eficiência global, se fossem revisados e acrescentados parâmetros de consumo energético e de insumos, por exemplo, e seguindo os modelos de indicadores de desempenho de destaque no âmbito internacional, conforme apontado no item anterior desse estudo. Assim, esses índices seriam capazes de avaliar de forma equâni- 
me o desempenho de duas estações e possibilitar a comparação. Essa característica é ainda mais interessante na ótica do empreendedor, que poderá avaliar sua estação não apenas sob a perspectiva ambiental, mas também sob a perspectiva de eficiência operacional e econômica, levando em conta critérios de custo-benefício.

Um ponto relevante a ser destacado das referidas normas é a previsão da divulgação anual dos indicadores na Intranet do INEA. É interessante perceber que tal característica dialoga diretamente com o princípio da informação, um dos princípios do direito ambiental.

O maior objetivo do princípio da informação no direito ambiental é, efetivando o direito à informação, permitir aos indivíduos a participação ativa nas questões relativas ao meio ambiente. [...] E essa participação pode se dar tanto no contexto particular ou individual, com o intuito de diminuir a degradação ambiental, quanto na esfera pública, impondo às autoridades administrativas e judiciais uma atuação adequada e efetiva, através dos meios legais disponíveis (Gomes; Simioni, 2014, p. 129).

A divulgação dos resultados para a população também está relacionada com o princípio fundamental de transparência das ações - princípio IX - estabelecido na Lei $\mathrm{n}$ 은 $11.445 / 2007$, que estabelece os princípios fundamentais para os serviços públicos de saneamento básico. Outro ponto que é importante mencionar é o disposto no art. 23, X da Lei, que trata da necessidade de disponibilização de informação sobre o saneamento, a saber: "padrões de atendimento ao público e mecanismos de participação e informação" (grifo nosso).

Por outro lado, ao contrário do que o INEA realiza com os demais índices, como o Índice de Qualidade da Água, que avalia a qualidade dos rios, não há uma divulgação periódica dos resultados das ETE. Na verdade, não há registros no banco de dados do site do INEA, de qualquer publicação dos resultados relativos aos índices das estações de tratamento de efluentes do estado.

\section{Comparação entre IQE e o IQETDI}

Realizou-se uma comparação entre os índices criados pelo INEA, cuja síntese está disposta no Tabela 2.

Pela comparação entre os dois indicadores IQE e IQETDI nota-se, de maneira geral, que o IQE leva em consideração mais critérios do que o IQETDI. Além disso, os critérios atrelados à localização da estação, ao plano de contingência e ao estado de manutenção dos equipamentos são avaliados apenas no IQE. No entanto, essa diferença na avaliação é questionável, pois tais critérios não têm relação com a origem do efluente ou, ainda, com alguma legislação específica de ETE doméstico.

Observa-se também uma significativa diferença nos pesos atribuídos aos critérios em comum entre os índices. O critério que avalia o número de violações cometidas no IQETDI tem o dobro do peso do IQE; o mesmo ocorre no critério sobre acesso ao medidor de vazão e destinação adequada de resíduos.

Tais diferenças não se justificam, pois os instrumentos legais que regulam esses itens não as respaldam. Por exemplo, a destinação inadequada de resíduos é penalizada pela Lei Estadual no 3467/2000 (Rio de Janeiro, 2000), sem distinção sobre sua origem (industrial ou doméstica).

Apesar de ambos os índices terem como objetivo avaliar o desempenho de ETE, há uma distinção em relação à tipologia do efluente tratado (doméstico ou industrial). No entanto, as diferenças encontradas entres os dois índices não estão relacionados à tipologia do efluente a ser tratado, ou seja, não configuram uma avaliação ou uma abordagem distinta. A diferença se concentra apenas na quantidade e nos pesos atribuídos em alguns critérios.

Os critérios que são avaliados apenas no IQE não são particulares de estações de efluentes domésticos; ao contrário, também poderiam ser avaliados em estações de efluentes industriais. Dentre esses critérios, pode-se citar a presença de sistema de desinfecção e os materiais sedimentáveis e flutuantes no efluente tratado.

Vale apontar um erro na avaliação do parâmetro odor característico no efluente, nos dois índices, pois a pontuação é dada quando há presença de odor característico. No entanto, não faz sentido penalizar o efluente inodoro e bonificar o efluente que apresente odor.

Dessa forma, não fica claro o objetivo do órgão em instituir dois indicadores distintos, sendo que teria sido possível criar apenas um que avaliasse as ETE, sejam eles de origem doméstica ou industrial. Ademais, como percebido pela análise, a diferença na avaliação da estação de tratamento quanto à tipologia do efluente não é encontrada nos indicadores propostos em outros países (LNEC, SWWA e AWWA, por exemplo). Sendo assim, um único indicador que avaliasse ETE poderia ser criado, englobando não só dos critérios dispostos no nos dois atuais índices, mas como também critérios capazes de avaliar a eficiência energética e da gestão de insumos.

Outro ponto de grande significância, além da revisão dos critérios, seria a revisão dos pesos a eles aplicados, podendo ser realizada uma pesquisa com atores impor- 
Tabela 2. Comparação entre os critérios do IQE e IQETDI

\begin{tabular}{|c|c|c|}
\hline Critério & IQE & IQETDI \\
\hline Proximidade de Núcleos Habitacionais & $\mathrm{x}$ & \\
\hline Zoneamento Municipal & $\mathrm{x}$ & \\
\hline Status Operacional das unidades que compõem o sistema & $\mathrm{x}$ & $x$ \\
\hline $\begin{array}{c}\text { Estado de conservação da infraestrutura civil (Gradeamento, caixa de areia, decantadores primários, secundário, } \\
\text { lagoas etc.) }\end{array}$ & $\mathrm{x}$ & $\mathrm{x}$ \\
\hline Estado de manutenção das máquinas e equipamentos operacionais & $\mathrm{x}$ & \\
\hline Cor do efluente tratado (Ausência de cor - Translúcido) & $\mathrm{x}$ & $\mathrm{x}$ \\
\hline Automação da ETDI & & $\mathrm{x}$ \\
\hline Materiais sedimentáveis no efluente tratado (teste de 1 hora em "Cone Imnhoff") & $\mathrm{x}$ & \\
\hline Materiais flutuantes no efluente tratado (Ausente) & $\mathrm{x}$ & \\
\hline Odor característico (Perceptível fora dos limites do ETE) & $\mathrm{x}$ & $x$ \\
\hline Vinculação ao Programa de Auto Controle de Efluentes Líquidos & $\mathrm{x}$ & $\mathrm{x}$ \\
\hline Atendimento à frequência de monitoramento pelo órgão ambiental competente & $\mathrm{x}$ & $x$ \\
\hline Atendimento aos parâmetros estabelecidos & $\mathrm{x}$ & $x$ \\
\hline Número de violações aos padrões de lançamento vigentes nos últimos 3 meses & $\mathrm{x}$ & $\mathrm{x}$ \\
\hline Armazenamento de produtos químicos necessários à operação da ETE/ETDI & $\mathrm{x}$ & $\mathrm{x}$ \\
\hline Possui medidores de vazão acessível a fiscalização & $\mathrm{x}$ & $\mathrm{x}$ \\
\hline Possui unidade de tratamento prévio de lodo em operação & $\mathrm{x}$ & $x$ \\
\hline Destinação adequada dos resíduos com Manifesto para empresa licenciada & $\mathrm{x}$ & $\mathrm{x}$ \\
\hline Possui certificado de ART do operador da ETE/ETDI & $\mathrm{x}$ & $\mathrm{x}$ \\
\hline Possui outorga de lançamento de efluente tratado & $\mathrm{x}$ & $x$ \\
\hline Operação de acordo com o material aprovado pelo órgão Ambiental & $\mathrm{x}$ & $x$ \\
\hline Plano de inspeção e manutenção & $\mathrm{x}$ & $\mathrm{x}$ \\
\hline Plano de contingência & $\mathrm{x}$ & \\
\hline Possui sistema de reaproveitamento de Biogás & $\mathrm{x}$ & \\
\hline Possui sistema de desinfecção & $\mathrm{x}$ & \\
\hline Faz reuso do efluente tratado & $\mathrm{x}$ & $\mathrm{x}$ \\
\hline Credenciamento do laboratório que realiza análises do efluente & $\mathrm{x}$ & $\mathrm{x}$ \\
\hline Elaborou inventário de emissões de gases de efeito estufa no exercício do ano anterior & $\mathrm{x}$ & \\
\hline $\begin{array}{l}\text { Dados de análises dos parâmetros de controle da operação da ETDI (oxigênio dissolvido no tanque de aeração, } \\
\text { idade do lodo, dentre outros) }\end{array}$ & & $\mathrm{x}$ \\
\hline
\end{tabular}

tantes da área (analistas do órgão, instituições acadêmicas e empresas do ramo), conforme o proposto nos estudos realizados por Sperling e Sperling (2013), Barros (2013) e de Paula (2013).

\section{O licenciamento ambiental no estado do Rio de Janeiro e os indicadores de desempenho}

O estado do Rio de janeiro tornou público o seu novo Sistema Estadual de Licenciamento e Controle Ambiental (SELCA) por meio da publicação do Decreto Estadual no 46.890, de 23 de dezembro de 2019, que revogou o antigo Sistema de Licenciamento Ambiental (SLAM). O atual sistema passou por consulta pública e teve como principal viés a simplificação do procedimento de licenciamento ambiental, em consonância com o preconizado na última versão do Projeto de Lei no 3.729/2004 (Brasil, 2004), de autoria do deputado federal Kim Kataguiri, em tramitação no Congresso Federal.

O Decreto no 46.890/2019, em seu art. 13, estabelece:

O licenciamento e os demais procedimentos de controle ambiental levarão em conta indicadores de desempenho do empreendimento ou atividade (...) com vistas à efetividade na tutela do meio ambiente ecologicamente equilibrado e ao desenvolvimento econômico e social do estado do Rio de Janeiro. (grifo nosso).

O texto normativo expressa a importância de indicadores de desempenho na avaliação e no controle ambiental de empreendimentos e atividades, especialmente no âmbito do licenciamento ambiental. Nesse caminho, o 
SELCA coloca a responsabilidade de estabelecer indicadores para o próprio órgão ambiental estadual, conforme o art. 14: "O órgão ambiental competente buscará estabelecer, como regra geral, a adoção de indicadores de desempenho, ao invés de meios para atingi-los, em respeito ao princípio da livre iniciativa".

Nesta conjuntura, nota-se a necessidade de o INEA reavaliar e promover a aplicação dos seus índices de avaliação da qualidade de ETE, IQE e IQETDI, visto que estes adquiriram uma maior importância legal com a publicação do SELCA no estado do Rio de Janeiro.

Outro ponto interessante que surge com o novo sistema de licenciamento ambiental estadual é a dependência da avaliação de critérios de sustentabilidade para a fixação dos tempos máximo e mínimo da vigência das licenças ambientais. Os referidos critérios são divididos em quatro grupos: gestão ambiental; produtos e resíduos; eficiência hídrica, energia e emissões; e conservação da natureza.

O indicador de desempenho de ETE é uma excelente ferramenta capaz de avaliar a eficiência da gestão dos insumos e energética. Igualmente, vale destacar que o indicador possibilita a melhoria contínua da estação, considerando que na sua aplicação são identificados pontos de melhoria e correção (ADASA, 2016; ERSAR, 2020).

Para o empreendedor é atrativo implementar este indicador, uma vez que pode contribuir para que a validade da licença ambiental de sua atividade alcance o prazo máximo estabelecido no referido decreto. Vale ressaltar que em relação à licença de operação (LO), por exemplo, a diferença entre o prazo mínimo e o máximo é de seis anos. $O$ mesmo acontece na licença ambiental unificada (LAU), cujo prazo mínimo é de seis anos e o máximo é de 12 anos, ou seja, uma diferença também de seis anos.

Ainda sobre o aspecto da renovação das licenças ambientais, o Decreto dispõe, em seu art. 31, que "nos casos em que o Relatório de Auditoria de Controle Ambiental for aprovado pelo INEA, sem que sejam detectadas não conformidades, a renovação da Licença Ambiental poderá se dar de forma expedita, conforme disposto em regulamento".

Com isso, a Auditoria de Controle Ambiental, regulamentada pela Diretriz INEA 056-R3 (INEA, 2010), ganha maior destaque no licenciamento ambiental, tornando-se uma oportuna ocasião para aplicação e avaliação dos IQE ou IQETDI pelos auditores, a fim de respaldar e fundamentar, de forma analítica, o relatório final da auditoria. Vale ressaltar que um dos grandes empecilhos enfrentados pelo órgão ambiental para aplicação destes indicadores é o reduzido número de técnicos, de acordo com o depoimento da analista responsável pelo serviço de licenciamento de ETE do INEA entrevistada. Com isso, haveria a aplicação do indicador ao menos uma vez ao ano nas estações de tratamento de efluentes de empreendimentos fiscalizados e licenciados pelo estado.

O Projeto de Lei $n^{\circ} 3729 / 2004$ que promulga a Lei Geral do Licenciamento Ambiental, em seu último texto, em tramitação no Congresso, estabelece, no art. 15, que empreendimentos que tiverem adotado medidas que possibilitem alcançar resultados mais rigorosos do que os critérios estabelecidos pela legislação ambiental vigente poderão desfrutar de condições especiais, tais como priorização nas análises e dilatação dos prazos de renovação de licenças ambientais. Assim sendo, a implementação de indicadores de desempenho por parte dos empreendedores está em consonância com o preconizado no texto atual deste projeto de lei.

\section{CONCLUSÃO}

Os indicadores de desempenho ambiental são instrumentos que contribuem para a preservação ambiental, uma vez que favorecem uma avaliação mais criteriosa e, no geral, estimulam a busca por resultados de mais alto nível. Esses instrumentos estão de acordo com o direito fundamental preconizado no art. 225 da Constituição Federal de 1988 que declara que todo indivíduo tem direito à um ambiente ecologicamente equilibrado.

Em relação à avaliação de ETE, os indicadores de desempenho podem oferecer uma contribuição de extrema relevância como critério de avaliação, benchmarking de boas práticas, fundamentação em pareceres técnicos de órgãos ambientais e, ainda, no Licenciamento Ambiental conforme as legislações ambientais do estado do Rio de Janeiro e em tramitação no Congresso Nacional atuais têm apontado.

Nesse contexto, o INEA apresentou indicadores de desempenho de ETE que possuem um elevado potencial de aplicação e obtenção de informações e resultados sobre estações de uma forma clara e objetiva, podendo ser divulgado para população em geral, facilitando o acesso a informações demasiadamente técnicas e de difícil compreensão por leigos.

Por outro lado, ainda cabem aperfeiçoamentos, como a unificação dos dois indicadores (IQE e IQETDI) gerando um único indicador de desempenho destinado à avaliação de ETE (domésticos ou industriais). Outro ponto importante é que este indicador único proporcione avaliação da ETE nas seguintes dimensões: operação e 
infraestrutura, qualidade (efluente e lodo), gestão de insumos e aspectos econômico-financeiros, conforme observado nos indicadores de desempenho internacionais. Além de uma revisão dos pesos dos critérios através de uma consulta com atores importantes do setor (stakeholders: analistas do órgão, instituições acadêmicas e empresas do ramo), conforme realizados em estudos de proposição de indicadores de desempenho na área de saneamento.

\section{REFERÊNCIAS}

Agência Reguladora de Águas, Energia e Saneamento Básico do Distrito Federal - ADASA (2016). Resolução no 008, de 04 de julho de 2016. Diário Oficial do Distrito Federal, 128, 6 jul. 2016.

Balmér, P.; Hellström, D. (2012), Performance indicators for wastewater treatment plants. Water Science and Technology 65, 7, 1304-1310. https://doi.org/10.2166/ wst.2012.014

Barros, I. P. A. F. (2013). Proposta de um sistema de indicadores de desempenho para avaliação de Estações de Tratamento de Esgotos do Distrito Federal. Dissertação de Mestrado, Universidade Federal de Minas Gerais, Minas Gerais. Disponível em: www.smarh.eng.ufmg.br/ defesas/804M.PDF (acesso em: 10 ago. 2019).

Bertoletti, E. (2015). A Presunção Ambiental e a Ecotoxicologia Aquática, Ministério Público Federal, disponível em: www.mpf.mp.br/atuacao-tematica/ccr4/ dados-da-atuacao/projetos/qualidade-da-agua/boletim-das-aguas/edicao-2015/a-presuncao-ambiental-e-a-ecotoxicologia-aquatica-1 (acesso em: 26 abr. 2020).

Brasil. Congresso Nacional. Subemenda substitutiva global de plenário. Projeto de Lei da Câmara n.o 3.729 de 2004. Autoria: Deputado Kim Kataguiri (2019), disponível em: www2.camara.leg.br/atividade-legislativa/comissoes/grupos-de-trabalho/56a-legislatura/licenciamento-ambiental/documentos/outros-documentos/texto-base-4a-versao-apresentado-em-08-08.2019 (acesso em: 21 mar. 2020).

Brasil. Lei 11.445, de 5 de janeiro de 2007. Diário Oficial da União, 8 jan. 2007. Disponível em: www.planalto.gov. br/ccivil_03/_ato2007-2010/2007/lei/l11445.htm (acesso em: 21 mar. 2020).

De Paula, R. L. (2013). Metodologia para avaliação de desempenho operacional de estações de tratamento de esgotos, utilizando métodos multiobjetivo e indicadores. Dissertação de Mestrado, Universidade de Brasília, Brasília, DF, Brasil. Disponível: https://repositorio.unb.br/ bitstream/10482/14718/1/2013_ReuelLopesDePaula. pdf. (acesso em: 23 mar. 2020).
Entidade Reguladora de Águas e Resíduos de Portugal - ERSAR (2020). Guia Técnico 22 - Guia de Avaliação da Qualidade dos Serviços de Águas e Resíduos Prestados aos Utilizadores - 3a Geração do Sistema de Avaliação, Lisboa, 2020. Disponível em: www.ersar.pt/pt/publicacoes/publicacoes-tecnicas/guias (acesso em: 23 mar. 2020).

Gomes, R. N.; Simioni, R. L. (2014). A aplicação do princípio ambiental da informação no direito brasileiro na forma de confiança e risco em Niklas Luhmann. Revista Direito Ambiental e Sociedade 4, 2, 117-136. Disponível em: www.ucs.br/etc/revistas/index.php/direitoambiental/article/view/3341/2255 (acesso em: 10 ago. 2019).

Instituto Estadual do Ambiente - INEA (2010). Diretriz para Realização de Auditoria Ambiental. Diretriz INEA 056-R3. Rio de Janeiro, RJ. Disponível em: http:// www.inea.rj.gov.br/wp-content/uploads/2019/11/DZ-0056.R-3.pdf. (acesso em: 10/03/2020)

Instituto Estadual do Ambiente - INEA (2015). Metodologia para cálculo do Índice de Qualidade de Operação de Estação de Tratamento de Esgoto (IQE), NOI/INEA-11, Rio de Janeiro, RJ.

Instituto Estadual do Ambiente - INEA (2015). Metodologia para cálculo do Índice de Qualidade de Operação de Estação de Tratamento de Despejos Industriais (IQETDI), NOI/INEA-14, Rio de Janeiro, RJ.

Instituto Estadual do Ambiente - INEA (2018). ATA da 425a Reunião Ordinária de Licenciamento Ambiental do Condir do dia 04/04/2018. Disponível em: www.inea. rj.gov.br/cs/groups/public/@inter_pres_aspres/documents/document/zwew/mtu0/ edisp/inea0154207.pdf (acesso em: 10 ago. 2019).

Instituto Estadual do Ambiente - INEA (2018). Parecer Técnico de Licença de Operação $n^{\circ} 13 / 18$ fl 1/7, Sistema de Consulta Unificada de Processo do INEA. Disponível em: http://sistemas.inea.rj.gov.br/meioambiente/ arquivos/licenciamento/parecer/164636/PARECER\%20 GELANI\%20N\%C2\%B0\%2013-18\%20-INDEF\%20LO\%20 IN001546-ETIG\%20CEDAE..pdf (acesso em: 10 ago. 2019).

Instituto Trata Brasil (2019). Internações de doenças por veiculação hídrica no Brasil. Disponível em: www.tratabrasil.org.br/blog/2019/05/21/internacoes-de-doencas-por-veiculacao-hidrica-no-brasil/ (acesso em: 10 ago. 2019).

Lindtner, S., Schaar, H., Kroiss, H. (2008). Benchmarking of large municipal wastewater treatment plants treating over 100,000 PE in Austria. Water Science and Technology 50, 7, 1487-1493.

Melo, J. F. M.; Sousa, A. F. (2014). Indicadores de desempenho ambiental no setor público: uma análise nos gas- 
tos ambientais e no desempenho verde de municípios, artigo apresentado nos Anais do XVI Encontro Internacional sobre Gestão Empresarial e Meio Ambiente - ENGEMA, São Paulo, SP, Brasil, 2014. Disponível em: www. engema.org.br/XVIENGEMA/18.pdf, (acesso em: 21 mar. 2020).

Ministério do Meio Ambiente - MMA. (2019). Indicadores Ambientais. Disponível em: www.mma.gov.br/informacoes-ambientais/indicadores-ambientais (acesso em: 10 ago. 2019).

Piza, F.J.T.; Paganini, W.S. (2006). Uma proposta de indicadores. In Galvão Júnior, A.C.; Silva, A.C. Regulação: indicadores para a prestação de serviços de água e esgoto. 2 ed. Fortaleza: Expressão Gráfica. p. 204.

Raschle, L. H. (2013). Avaliação de desempenho para estações de tratamento de efluentes industriais. Disser- tação de mestrado, Universidade do Estado do Rio de Janeiro, Rio de Janeiro, RJ. Disponível em: www.peamb. eng.uerj.br/trabalhosconclusao/2013/Lilian-Dissertacao.pdf (acesso em: 21 mar. 2020).

Rio de Janeiro (2000). Lei no 3.467, de 14 de setembro de 2000. Diário Oficial do Estado do Rio de Janeiro, Rio de Janeiro, 15 set. 2000.

Rio de Janeiro (2019). Decreto no 46.890, de 23 de dezembro de 2019. Diário Oficial do Estado do Rio de Janeiro, Rio de Janeiro, 24 dez. 2019, Seção 1, p. 5.

Sperling, T. L. V.; Sperling, M. V. (2013). Proposição de um sistema de indicadores de desempenho para avaliação da qualidade dos serviços de esgotamento sanitário. Engenharia Sanitária e Ambiental 18, 4, 313-322.

Recebido: 02 jul. 2020

Aprovado: 17 ago. 2020

DOI: $10.20985 / 1980-5160.2020 . v 15 n 2.1659$

Como citar: Ramos Junior, W. (2020). Análise crítica dos indicadores de desempenho de estações de tratamento de efluentes do órgão ambiental estadual do Rio de Janeiro. Revista S\&G 15, 2, 181-189. https://revistasg.emnuvens. com.br/sg/article/view/1659 